FERMILAB-Conf-87/149

\title{
Channeling and Dechanneling at High Energy*
}

\author{
Richard A. Carrigan, Jr. \\ Fermi National Accelerator Laboratory \\ P.O. Box 500, Batavia, Illinois 60510
}

September 30, 1987

*Presented at the 12th International Conference on Atomic Collisions in Solids, Okayama, Japan, October 12-16, 1987 


\title{
CHANNELING AND DECHANNELING AT HIGH ENERGY
}

\author{
Richard A. Carrigan, Jr. \\ Fermi National Accelerator Laboratory
}

Batavia, IL 60510

September 30,1987

Abstract

The possibility of using channeling as a tool for high energy particle physics has now been extensively investigated. Bent crystals have been used as an accelerator extraction element and for particle deflection. Applications as accelerating devices have been discussed but appear remote. The major advantage in using a bent crystal rather than a magnet is the large deflection that can be achieved in a short length. The major disadvantage is the low transmission. A good understanding of dechanneling is important for applications. 


\section{Channeling at High Energy}

The phrase "high energy" suggests that there is something different about channeling in that regime or that one can define a "high" energy. Actually the basic channeling process behaves very much the same at $0.5 \mathrm{MeV}$ as it does at $0.5 \mathrm{TeV}$, gracefully spanning more than a range of a million in energy.

What then, defines high energy channeling? one feature might be the easy observation of the "donut" phenomenon, the tendency of particles beyond the critical angle to spiral around an axis. This phenomenon was observed in the seventies in the early high energy channeling experiments at $\mathrm{CERN}^{1}$ and at Fermilab ${ }^{2}$. Another could be the easy observation of heavy negative particle channeling via transmission measurements 1,2 . A third might be the fact that characteristic beam angular distributions have become larger than the critical angle so that dechanneling estimates must start with the entire channel phase space populated rather than having the beam lie very close to the axis or plane. Finally, "high" energy could be where it becomes practical to bend a crystal and thereby deflect or focus particles with channeling. This happens over a range of energies, 30-50 MeV for the SIN muon decay blocking measurements ${ }^{3}, 1 \mathrm{GeV}$ for the interesting Leningrad volume channeling experiments ${ }^{4}$, and $800 \mathrm{GeV}$ for the recent Fermilab NE crystal septum ${ }^{5}$. 
Planar dechanneling measurements in silicon illustrate just how well channeling theory works over this range of energies. Theoretical interpolation over this span must include phase space effects, the transition to relativistic kinematics, the possibility of defect dechanneling at very high energy even in extremely good silicon, and the behavior of logarithmic terms in multiple scattering. This can be done with:

$$
L(p)=L\left(p_{0}\right) *\left(p v / p_{0} v_{0}\right) * A_{V} / A_{v}
$$

Here $L(p)$ is the dechanneling length, $p$ and $v$ are the momentum and velocity of the particle, and $L\left(p_{0}\right)$ is a constant that is equivalent to a dechanneling length at a particular momentum. $A_{V}$ is a factor that incorporates the effect of phase space on dechanneling. It is 1.0 in the $\mathrm{MeV}$ regime and 0.55 in the $\mathrm{GeV}$ region when the channel is uniformly populated. Figure 1 shows these dechanneling lengths measured with many different techniques over the entire energy range ${ }^{6}$. In general the information is consistent to $20 \%$ and also agrees with theory at the same level. This agreement extends to extrapolation to other crystal orientations as well as to some other elements. It is harder to infer such nice behavior for the axial dechanneling. This may be in part because temperature effects are larger. The most obvious problem is tungsten. There the experiments of Davies et al. ${ }^{7}$ show almost no energy dependence for axial dechanneling in the $\mathrm{MeV}$ 
regime. It is unlikely that this is a fundamental problem. More likely it has to do with questions of crystal perfection. Note that the highest energy for which dechanneling measurements in tungsten have been made is $83 \mathrm{MeV}^{8}$. For tungsten crystals of currently-available quality, it might be possible to observe axial channeling up to the $10 \mathrm{GeV}$ region.

Negative particle channeling and dechanneling is a subject that could receive more attention. Theoretical treatments are harder because the beam particles spend much more time in the vicinity of the atomic ions and questions of quasi-channeling become important. Theoretical results differ, depending to some extent on how quasi-channeling is treated $^{9}$. Recent experimental results for electron dechanneling are also shown in Figure 1. In general these results are not particularly consistent. As an example, the results of Berman et al.10 do not exhibit a linear energy dependence. Note that result for positron dechanneling does agree at the $50 \%$ level.

There is very little experimental information on heavy negative particle channeling. Negative particle dechanneling lengths in the $100 \mathrm{GeV}$ regime ought to be long enough so that it should be possible to do reasonable dechanneling measurements. Such experimental information could provide a significant test of theory.

Bending dechanneling measurements 11 so far have only been good enough to show that the broad understanding of the process as set forth in theories such as Ellison ${ }^{12}$ and kudo ${ }^{13}$ is correct. 
It would be useful to have a full theoretical treatment that related bending dechanneling to ordinary dechanneling. Since the mean trajectory is shifted closer to atomic centers in a curved crystal, it should be possible to effectively design the dechanneling length in a bent crystal. Would this offer a new and unique tool for studying atomic charge distributions?

\section{Beam Deflection With Crystals At High Energy Accelerators}

Channeling in bent crystals has now been used several times at accelerators for both extraction and for secondary beam deflection. Aside from the challenging technical problems of providing very high quality crystals and aligning them properly to tens of microradians, application of the technique is limited by the transmission of the bent crystal. These factors are summarized in Table $I$.

The crystal transmission, E, for bending by a silicon plane is:

$$
E \approx E_{C}\left(1-p / 3 R_{m}\right) \exp \left(-s p_{0} / \lambda_{0} p\right) \Phi_{b} 50 / \Phi
$$

where $\Phi$ refers to the emittance of the beam or the admittance of the crystal, $R_{m}$ is the minimum radius of curvature in $c m, p$ is the momentum in $\mathrm{GeV} / \mathrm{C}, \mathrm{E}_{\mathrm{C}}$ is the surface acceptance, $s$ is the length of the crystal, and $p_{0}$ is the momentum for the dechanneling length $\lambda_{0}$. The arguments for this formula are developed more 
fully elsewhere ${ }^{14}$.

Up until now bent crystals have been used or considered for four major areas of applications; extraction from accelerators, secondary beam optics, and deflection and spin measurements of short-lived particles. These are summarized in Table II.

\subsection{Extraction}

The bent crystal technique has been used successfully at Dubna to extract beam from the accelerator at energies up to 7.5 $\mathrm{GeV}^{15}$. The short length of the crystal offers an interesting advantage over ordinary electromagnetic devices because of the constraints of the accelerator lattice. At Dubna the extraction transmission was $0.01 \%$, limited mainly by the very large beam size. At higher energies this should be less of a limitation.

A novel but very speculative extraction possibility is to use a crystal to bend a proton beam toward the center of the earth and then use the deflected protons to form a neutrino beam ${ }^{14}$. Following a suggestion of De Rujula et al.16, such a neutrino beam could be used to explore the density of the earth . A beam from the Tevatron would only be sufficient to demonstrate this possibility.

Crystals have also been discussed as extraction elements for the proposed $20 \mathrm{TeV}$ superconducting super Collider (SSC) ${ }^{17}$. For the SSC, a $10 \mathrm{~cm}$ long crystal could be used to extract a substantial parasitic beam with up to $90 \%$ efficiency. 
Note that radiation damage does not seem to be a limitation on extraction. No substantial degradation of channeling properties has been observed 18 below fluences of $10^{18} / \mathrm{cm}^{2}$. Observable dechanneling from damage due to proton beams in the $\mathrm{GeV}$ range only starts to be seen for fluences in the $10^{19}$ to $10^{20} / \mathrm{cm}^{2}$ range.

\subsection{Beam Deflection}

At Fermilab bent crystals have been used as beam deflection elements in several secondary beams. The first such application was in M-Bottom where a crystal septum was used to raise the beam energy from 225 to $400 \mathrm{GeV}^{19}$. Beam transmission was $0.03 \%$. The transmission predicted by (2) was $0.15 \%$. More recently a bent crystal has been used as a beam throttle in NE operating at 800 $\mathrm{Gev}^{5}$. The crystal reduced the beam after a high intensity experiment so it could be used downstream in a low intensity emulsion experiment. Here the observed beam transmission was $0.05 \%$, about a factor of six less than expected.

At this point the factor of five to six difference between the observed and predicted transmission is something of a mystery. It might be due to some combination of misalignment of the body of the crystal relative to the planar direction, the onset of problems with dislocations or interstitial imperfections, overestimation of the surface acceptance, or an improper understanding of the crystal beam optics. 


\subsection{Short-lived Particles}

Another potential application of bent crystals is for deflecting short-lived particles. It should be possible to deflect these charm and beauty particles on the order of fifty milliradians in the short distance before they decay. This is well out of the forward production cone. The technique could give a substantially-enriched sample of short-lived particles if the long-lived particles in the same channel continue around the bent crystal. As a result it might make it possible to provide an inclusive trigger for charm decays. For planar geometries, the angular acceptance is on the order of $1 \%$ of the production distribution. $\operatorname{sun}^{20}$ estimates that one could obtain several hundred charm triggers per hour in a typical experiment. Dechanneling of long-lived particles as well as production particle interactions may constitute serious backgrounds.

Pondrom 21 has noted that the average electric field in a curved crystal channel transforms into a magnetic field in the rest frame of a particle moving in the channel. A spinning particle will precess around that magnetic vector. Effective fields in a bent crystal are sufficient to precess a magnetic moment several radians in $1 \mathrm{~cm}$. For a polarized process it should be possible to measure the precession in the same way as has been done for strange particles. $\mathrm{Kim}^{22}$ has discussed these possibilities in detail. 


\section{Exotic Accelerators}

Normal accelerating structures, such as an rf linac, operate with accelerating gradients of $0.2-1.0 \mathrm{MeV} / \mathrm{cm}$. Because of the length, this is a substantial limitation when one considers the possibility of linacs in the $1000 \mathrm{GeV}$ range.

If a shorter linac is desired, the gradient can be increased up to the limit of electrical breakdown in the accelerator walls. since the breakdown-limiting gradient increases with frequency, one approach is to increase the rf frequency. The power density per unit length is proportional to the square of the gradient so that power requirements become very high.

Several possibilities have been considered for drastically increasing accelerating gradients. One is to somehow directly use a laser to accelerate particles. Another is to set up a longitudinal electron plasma traveling wave.

Laser acceleration should be able to give accelerating gradients up to $5 \mathrm{GeV} / \mathrm{cm}$. Unfortunately the electric field is normally transverse to the direction of the laser, making it difficult to get longitudinal acceleration and leading to transverse acceleration with concomitant radiation losses.

\subsection{Plasma Accelerators}

For plasmas, it is relatively easy to set up a longitudinal 
accelerating field. Plasmas also have the feature that they are immune to electrical breakdown.

Some driver is necessary to excite the plasma wave. In thinking about these accelerators, it is well to keep the problem of the driver to generate the plasma wave separated from the subject of the accelerator. The plasma wave driver here is the analog of the klystron in a conventional rf linac. One possibility for a driver is to use a second high-current, lowenergy particle beam. This is the wake-field accelerator concept. A second is to use a laser or lasers (the beat-wave accelerator). In any case it will turn out that this driver will have to deposit very large amounts of energy in the plasma medium.

The maximum gradient for a plasma can be found by using Poisson's equation and taking the case where all the plasma electrons are removed at points of rarefaction for the plasma wave 23 . Substituting the plasma frequency $\omega_{p}=\left(4 \pi n_{0} e^{2 / m_{e}}\right)^{1 / 2}$ into Poisson's equation gives

$$
\mathrm{eE}_{\max } \approx 0.97 \sqrt{\mathrm{n}_{0}} \mathrm{eV} / \mathrm{cm},
$$

where $E_{\max }$ is the so-called cold wavebreaking field. Here $\mathrm{n}_{0}$ is the equilibrium electron density. In a gas $n_{0}$ could be up to $10^{18} / \mathrm{cm}^{3}$ giving rise to a gradient of $1 \mathrm{GeV} / \mathrm{cm}$. Clearly this is a significant step forward.

Why, then, aren't there plasma wave accelerators? The theory is quite well understood. Practical accelerating systems have 
been extensively modeled 23 , and acceleration has been demonstrated in plasmas $1-10 \mathrm{~mm}$ long $^{24}$.

However there are problems. One is phase coherence. Once the particle has fallen into the trough of the plasma wave it no longer accelerates. In accelerator terms, the phase velocity of the plasma wave is less than the speed of light so that the relativistic accelerated particles will soon outrun the wave. This can be obviated by the so-called surfatron ${ }^{25}$ where the particle beam is skewed in direction relative to the plasma wave. Another problem is that the efficiency of presently-conceived plasma wave drivers is much less than the klystrons used in conventional rf linacs.

Typically for the laser beat wave scheme for plasma wave generation the accelerating wave pulse will be about 50 oscillations long. In a gas the number of oscillations goes as $\sqrt{\mathrm{M} / \mathrm{m}}$ where $\mathrm{M}$ is the lattice atom mass and $\mathrm{m}$ is the electron mass. This length is set by the fact that a conventional gaseous plasma becomes turbulent after some number of oscillations due to electron-ion instabilities.

Characteristically the plasma angular frequency in a metal is $10^{16} \mathrm{~Hz}$. The plasma decay time is $10^{-14} \mathrm{~s}$ because of interband transitions, so that the plasma wave is again on the order of 100 oscillations long. The beam cross section must also be quite small. If the plasma accelerator is operating as a near field accelerator like a conventional linac, then the transverse scale is set by the plasma wavelength so that it will be in the 
neighborhood of $10^{-4}$ to $10^{-5} \mathrm{~cm}$. For the evanescent case described later the transverse size will be similar but set by power considerations. This small cross section and short pulse length will limit the amount of beam that can be accelerated in any driver beam pulse.

There are other problems. Because of the turbulence, plasmas are naturally unstable so that all processes will be non-linear. This leads to problems both for accelerator beam focusing and chromaticity.

\subsection{Solid State Accelerators}

Forgetting these problems for the moment, it is interesting to examine the possibility of a solid state plasma accelerator. In a metal the density of the plasma can go up to $10^{22}$ electrons $/ \mathrm{cm}^{3}$ or about ten thousand times higher than a plasma in a gas. Since the gradient is proportional to $\sqrt{n}$, the equivalent gradient will be a factor of 100 higher or in the range of 100 $\mathrm{GeV} / \mathrm{cm}$.

For a solid, it is necessary to consider the nuclear interaction length and the radiation length in the material for the accelerated particles. In an amorphous solid the nuclear interaction length is typically $10-50 \mathrm{~cm}$ while the radiation length is $10-0.4 \mathrm{~cm}$. The nuclear interaction length constitutes a definite constraint on the peak energy. After several interaction lengths most of the particles in the beam will have suffered 
large angle collisions and left the acceptance phase space of the accelerating plasma wave. Similarly, the radiation length will limit the ability of a particle to gain energy.

A crystal aligned for channeling should ameliorate the impact of both of these factors. Nuclear interactions for channeled particles should be substantially reduced, since there are few nuclei, such as interstitials, in the channel. Normal radiation will also be modified. The maximum energy for this situation is approached when the radiative energy loss nears the energy gain from acceleration. Chen and Noble 26 have studied this and shown that the maximum energy is limited by channeling radiation to

$$
E_{\max } \approx(\mathrm{m} / \mathrm{M})^{2}(\Lambda \mathrm{G})^{1 / 2}\left\{\mathrm{G} /\left(\mathrm{z}^{3} * 100 \mathrm{GV} / \mathrm{cm}\right)\right\}^{1 / 2} 10^{5} \mathrm{TeV}
$$

where $m$ and $M$ are the mass of the beam particle and the mass of the proton respectively, $\Lambda$ is the dechanneling length per unit energy, $G$ is the accelerating gradient, and $z$ is the charge of the beam particle. This formula is based on using the radiation loss per unit length in a smooth focusing accelerator system 27 but could equally well be derived from channeling radiation theory. Characteristically this would limit the maximum energy for protons to $10^{5} \mathrm{TeV}$ and for positrons to $10 \mathrm{GeV}$.

on the other hand dechanneling now becomes a significant factor. The treatment of dechanneling must be handled slightly differently than normal since the particles are accelerating. In 
some sense this is the obverse of low energy dechanneling where a particle may lose all of its energy in the process of being dechanneled.

Chen and Noble 26 treat this by using a dechanneling length that scales with the total energy. They also introduce a normalized rms acceptance from accelerator theory:

$$
E_{\mathrm{Cn}}=(1 / 2) Y \mathrm{a} \psi_{\mathrm{C}}
$$

where $Y$ is the Lorentz factor, a is the axial channel radius, and $\psi_{C}$ is the critical angle. Note that this will scale as $\sqrt{E}$. They show that particles will remain channeled as they are accelerated provided $G \geq \Lambda^{-1}$.

A number of solid state accelerator concepts have been suggested. They are summarized in Table III. These include purely laser schemes such as the suggestion of Kanofsky ${ }^{28}$. His approach uses a laser transverse to the direction of the accelerator with a masking grating to eliminate phase reversal. Kanofsky noted that accelerating in a channeling direction could reduce interactions of the particle beam with the medium.

Several Russian groups have looked at various laser combinations. Nasonov 29 proposed setting up a static charge distribution in alkali-halide crystals by driving with optical phonons. This delivers a relatively modest gradient of $10 \mathrm{MV} / \mathrm{cm}$. Grishaev and Nasonov 30 have considered a system of two coupled lasers to produce a longitudinal wave. They estimate the accelerating 
gradient would be $10-100 \mathrm{MV} / \mathrm{cm}$. Note that both the lasers as well as the accelerated particle beam would have to be phase matched. Pisarev 31 has discussed the use of a transverse laser swept along the crystal to produce phase matching. He estimates a similar value for the accelerating gradient. The required power density is in the neighborhood of $10^{12} \mathrm{~W} / \mathrm{cm}^{2}$. Belotshitskii and Kumakhov 32 studied both the physical limitations of this process as well as the efficacy of channeling in ameliorating the problems. They estimate the required power density as $10^{15} \mathrm{~W} / \mathrm{cm}^{2}$. Clearly with power densities this high the process is unconsciously drifting into the plasma regime. Chen and Noble 26 appear to have been the first to look at the accelerating process in terms of a solid state electron plasma. With that recognition they can separate the nature of the driver source to create the plasma wave from the plasma acceleration mechanism. As noted earlier, the plasma wave can be stimulated by either a laser or a particle beam. For a particle beam there is an additional advantage in channeling since energy loss due to the driver may also be lowered. Chen and Noble find that required power drive densities are in the range of $10^{15}$ to $10^{19} \mathrm{w} / \mathrm{cm}^{2}$.

Recently Tajima and Cavenago 33 have proposed driving the plasma wave using Bormann anomalous transmission of x-rays. For Bormann anomalous transmission the crystal geometry is arranged so that the $\mathrm{x}$-rays go directly down a channel. They propose use of $40 \mathrm{keV} x$-rays and state that the scheme can give gradients of $1 \mathrm{GV} / \mathrm{cm}$ but requires power densities of $10^{19} \mathrm{~W} / \mathrm{cm}^{2}$. 
As noted earlier, there are several very severe problems with all of these schemes. One is the extremely high power density required. Belotshitskii and Kumakhov ${ }^{32}$ appear to have been the first to have considered crystal damage in detail. They note that crystal destruction takes place at a power density of $10^{12} \mathrm{~W} / \mathrm{cm}^{3}$ for nanosecond-long pulses. This corresponds to current densities of $10^{5} \mathrm{~A} / \mathrm{cm}^{2}$. Experimentally, crystal breaking is observed at $10^{13} \mathrm{~W} / \mathrm{cm}^{2}$ for nanosecond laser pulses. This is roughly related to the fracture threshold for thermal shock. The exact fate of the crystal for a given energy density will depend on such things as the relaxation time for converting plasmon energy to phonons. Clearly many of these schemes rely on power densities well beyond the crystal breaking limit. Of course one can ask if the acceleration process can be completed before the damage occurs. If it does, it might be possible to use a new crystal for each acceleration cycle.

A second problem is the very small phase space. Characteristically such a bucket in phase space would contain $10^{5}$ particles if crystal fracture is to be avoided. One observation is in order. For any colliding beam device it is not the number of particles in the bunch that is important but the bunch luminosity. In any case, the intensity for a solid state accelerator seems to be far away from practical devices.

With such severe limitations one may ask why the possibility of solid state accelerators is pursued at all. There are several answers. The very high accelerating gradients have a great deal 
to offer. As an example, it should be possible to accelerate short lived particles ${ }^{32}$. Much will be gained if any ways can be found to avoid the problems.

The second point is that the subject leads into a number of interesting new areas for channeling and the interaction of beams and solids in general. For example, it would be useful to understand in more detail just how the channeling process degraded as a crystal was shocked with energy densities of $10^{12}$ $10^{14} \mathrm{~W} / \mathrm{cm}^{2}$. Another interesting possibility is to look at the channeling-like behavior of particles in zeolite structures ${ }^{34}$ or highly-ordered pyrolitic graphite ${ }^{35}$ where the material effectively has channels somewhat larger than in ordinary crystals. Such materials might have much longer dechanneling lengths, thereby reducing beam and driver losses in the crystal.

\section{Fundamental Channeling Information Still Needed}

While bent crystal channeling has been applied in several situations at high energies it would be rash indeed to say that the future is bright. The requirements on crystals and alignment are extreme and transmissions are low.

Nevertheless, bent crystal channeling does offer some interesting and even unique features. As noted throughout this paper it is still desirable to have more information on high energy channeling in order to pursue these possibilities.

In the theoretical area it would be useful to have a 
flexible phenomenological theory rooted in a more fundamental diffusion picture. It would also be desirable to see more work investigating the impact of bending on ordinary dechanneling. Experimental information on positive planar channeling is quite good. As noted earlier, there is relatively little axial information. In particular, the picture for tungsten is confused. It is important to clarify the situation for tungsten since the confusion may indicate the onset of widely anticipated defect problems even in the MeV regime.

So far bending channeling measurements are essentially qualitative. They need to be put on a quantitative basis that would match the modest accuracy of the normal dechanneling measurements.

For crystal channeling accelerators it would be interesting to explore the possibilities of exotic crystals such as highlyoriented pyrolitic graphite as well as channeling in crystals that were on the point of vaporization.

\section{ACKNOWLEDGEMENTS}

The author would like to thank J. Ellison and R. Noble for many useful discussions. 
FIGURE

1. Behavior of the ordinary dechanneling length (1/e) for $\mathrm{Si}$ (110) as a function of kinetic energy. (The points in the $\mathrm{GeV}$ regime are plotted at momentum values since they are for mixed beams. There is only a small error in doing this.) Three curves are shown: the solid curve is based on the ohtsuki 36 diffusion length extrapolated for pions, the dashed curve for protons, and the double dashed curve is the empirical fit to all energies including the effects of a broadly illuminated channel in the $\mathrm{GeV}$ range ${ }^{6}$. In the MeV regime this fit lies on the ohtsuki line and is not shown. The dots are Campisano et al.37, the crosses are Feldman and Appleton ${ }^{38}$, the diamond is Davies et al. 39, the CERN point is Bak et al.40, and the Fermilab data is Forster 41 . Also shown are electron points $10,42,43$ and a positron point ${ }^{10}$. 
Table I TRANSMISSION LIMITS AT HIGH ENERGY

\section{Factor}

Angular Acceptance

Spatial Acceptance

Normal Dechanneling

Bending Dechanneling

Surface Acceptance

\section{Iimit}

Critical Angle

Must Bend Crystal

Typically one dechanneling length

Phase Space Ellipse
Typical Value

0.1 of Beam

Crystal $=0.5 \mathrm{~mm}$ Beam $=5 \mathrm{~mm}$

$35 \%$

$50 \%$

$67 \%$ 
Table II APPLICATIONS AT HIGH ENERGY

Location

Dubna

Fermilab M-Bottom

Fermilab M-Top

Fermilab N-East
Purpose

Extraction

Double beam energy

Low intensity proton beam

Beam throttle
Energy Transmission

$7.5 \mathrm{GeV} \quad 0.01 \%$

$400 \mathrm{GeV} \quad 0.03 \%$

$800 \mathrm{GeV}$ failed

$800 \mathrm{GeV} \quad 0.05 \%$ 
Table III SOLID STATE ACCELERATOR SCHEMES

\begin{tabular}{|c|c|c|c|c|}
\hline Type & Author & $\begin{array}{l}\text { Acc. } \\
\text { Grad. }\end{array}$ & $\begin{array}{l}\text { Driver } \\
\text { Density } \\
\left(\mathrm{W} / \mathrm{cm}^{2}\right)\end{array}$ & Problems \\
\hline $\begin{array}{l}\text { Masked Laser with } \\
\text { Dielectric }\end{array}$ & Kanofsky 28 & & & \\
\hline $\begin{array}{l}\text { Two Laser Scheme to Produce } \\
\text { Longitudinally Polarized } \\
\text { Waves }\end{array}$ & $\begin{array}{l}\text { Grishaev and } \\
\text { Nasonov } 30\end{array}$ & $\begin{array}{l}10-100 \\
\mathrm{MV} / \mathrm{cm}\end{array}$ & & $\begin{array}{l}\text { Phase matching of } \\
\text { lasers, lasers to } \\
\text { particles }\end{array}$ \\
\hline $\begin{array}{l}\text { Longitudinal static Polar- } \\
\text { ization, phase matching } \\
\text { by sweeping laser beam }\end{array}$ & Pisarev 31 & $40 \mathrm{MV} / \mathrm{cm}$ & $10^{12}$ & \\
\hline Inverse Cerenkov Effect & $\begin{array}{l}\text { Belotshitskii } \\
\quad \& \text { Kumakhov }\end{array}$ & $1 \mathrm{GV} / \mathrm{cm}$ & $1_{0} 15$ & $\begin{array}{l}\text { Destruction of the } \\
\text { Crystal? }\end{array}$ \\
\hline $\begin{array}{l}\text { Longitudinal Optical } \\
\text { Phonons in Alkali-Halide }\end{array}$ & Nasonov 29 & $10 \mathrm{MV} / \mathrm{cm}$ & & \\
\hline $\begin{array}{l}\text { Longitudinal Electron } \\
\text { Plasma Wave }\end{array}$ & $\begin{array}{l}\text { Chen and } \\
\text { Noble }\end{array}$ & $100 \mathrm{GV} / \mathrm{cm}$ & $10^{15}-10^{19}$ & \\
\hline $\begin{array}{l}\text { Plasma Acceleration Using } \\
\text { Bormann Anomalous } \\
\text { Transmission of X-rays }\end{array}$ & $\begin{array}{l}\text { Tajima and } \\
\text { Cavenago }\end{array}$ & $1 \mathrm{GV} / \mathrm{cm}$ & $10^{19}$ & \\
\hline
\end{tabular}


REFERENCES

1. S. K. Anderson, O. Fich, H. Nielsen, H. E. Schiott, E. Uggerhoj, G. Charpak, G. Petersen, F. Sauli, J. P. Ponpon, and P. Siffert, Nuclear Physics B167, 1 (1980).

2. R. A. Carrigan, Jr., B. L. Chrisman, T. E. Toohig, W. M. Gibson, I.-J. Kim, C. R. Sun, Z. Guzik, T. S. Nigmanov, E. N. Tsyganov, A. S. Vodopianov, M. A. Hasan, A. S. Kanofsky, R. Allen, J. Kubic, D. H. Stork, and W. B. Watson, Nuclear Physics B163, 1 (1980).

3. B. D. Patterson, p. 459 in Relativistic Channeling, eds. R. A. Carrigan, Jr., J. A. Ellison, Plenum, New York (1987).

4. V. M. Samsonov, p. 129 in Relativistic Channeling, eds. R. A. Carrigan, Jr., J.A. Ellison, Plenum, New York (1987).

5. S. I. Baker, R. A. Carrigan, Jr., R. L. Dixon, H. C. Fenker, R. J. Stefanski, J. S. Forster, R. L. Wijayawardana, and S. Reucroft, Nucl. Instr. and Meth. A248, 301 (1986).

6. R. A. Carrigan, Jr., Fermilab FN-454 (1987).

7. J. A. Davies, J. Denhartog, and J. L. Whitton, Phys. Rev. $165,345(1968)$.

8. W. Beezhold, T. W. L. Sanford, H. Park, J. O. Kephart, R. K. Klein, R. H. Pantell, B. L. Berman, and S. Datz, Bull. Am. Phys. Soc. 30, 374 (1985). W. Beezhold, private communication.

9. R. Wedell, p. 69 in Relativistic Channeling, eds. R. A. Carrigan, Jr., J. A. Ellison, Plenum, New York (1987).

10. B. L. Berman, J. O. Kephart, R. H. Pantell, S. Datz, H. Park, R. K. Klein, and B. A. Dahling, p. 239 in Relativistic Channeling, eds. R. A. Carrigan, Jr., J. A. Ellison, Plenum, New York (1987).

11. R. A. Carrigan, Jr. and W. M. Gibson, p. 61, coherent Radiation Sources, Eds. A.W. Saenz and H. Uberall, Springer-Verlag, Berlin (1985).

12. J. A. Ellison, Nucl. Phys. B206, 205 (1982).

13. H. Kudo, Nucl. Instr. Meth. 189, 609 (1981).

14. R. A. Carrigan, Jr., p. 339 in Relativistic Channeling, eds. R. A. Carrigan, Jr., J. A. Ellison, Plenum, New York (1987). 
15. V. V. Avdeichikov, et al., JINR Communication 1-84, Dubna (1984). English Translation: Fermilab FN-429 (1986).

16. A. De Rujula, S. L. Glashow, R. R. Wilson, and G. Charpak, Phys. Rep. 99, 341 (1983).

17. C. H. Sun, R. A. Carrigan, Jr., T. E. Toohig, and D. Neuffer, Proc. of the 1984 summer study on the Design and Utilization of the SSC, p. 483 (Snowmass-1984).

18. S. I. Baker, p. 391 in Relativistic Channeling, eds. R. A. Carrigan, Jr., J.A. Ellison, Plenum, New York (1987).

19. S. I. Baker, R. A. Carrigan, Jr., R. Schailey, T. E. Toohig, W. M. Gibson, I. -K. Kim, F. Sun, C. R. Sun, V. Tanikella, R. Wijayawardana, J. S. Forster, H. Hatton, I. V. Mitchell, and J. A. Ellison, Nucl. Instr. Meth. A234, 602 (1985).

20. C. R. Sun, p. 379 in Relativistic Channeling, eds. R. A. Carrigan, Jr., J. A. Ellison, Plenum, New York (1987).

21. L. Pondrom, Proc. of the 1982 DPF Summer School on Elementary Particle Physics and Future Facilities, eds. R. Donaldson, R. Gustafson, and F. Paige, Snowmass, CO (1982).

22. I. K. Kim, Nucl. Phys. B229, 251 (1983).

23. T. Katsouleas, C. Joshi, J. M. Dawson, F. F. Chen, C. Clayton, W. B. Mori, C. Darrow, and D. Umstadter, p. 63 in Laser Acceleration of Particles, C. Joshi and T. Katsouleas, eds., A. I. P. Conf. Proc. No. 130, New York (1985).

24. C. Joshi, et al. in Laser Acceleration of Particles, C. Joshi and T. Katsouleas, eds., A. I. P. Conf. Proc. No. 130, New York (1985).

25. T. Katsouleas and J. M. Dawson, Phys. Rev. Lett. 51, 392 (1983).

26. P. Chen and R. J. Noble, p. 517 in Relativistic Channeling, eds. R. A. Carrigan, Jr. and J. A. Ellison, Plenum, New York (1987).

27. B. W. Montague and W. Schnell, in Laser Acceleration of Particles, C. Joshi and T. Katsouleas, eds., A. I. P. Conf. Proc. No. 130, New York (1985).

28. A. Kanofsky, Rev. Sci. Instrum. 48, 34 (1977).

29. N. N. Nasonov, Sov. Tech. Phys. Lett. 6, 214 (1980). 
30. I. A. Grishaev and N. N. Nasonov, Sov. Tech. Phys. Lett. 3, 446 (1977).

31. A. F. Pisarev, Sov. Phys. Tech. Phys. 24, 456 (1979).

32. V. V. Belotshitskii and M. A. Kumakhov, Sov. Phys. Dokl. 24, 916 (1979).

33. T. Tajima and M. Cavenago, Inst. for Fusion studies (Texas) Preprint (1987).

34. J. M. Newsam, Science 231, 1093 (1986).

35. B. S. Elman, G. Braunstein, M. S. Dresselhaus, and G. Dresselhaus, T. Venkatesan, and B. Wilkens, J. Appl. Phys. 56, 2114 (1984), D. Schroyen, M.Bruggeman, I. Dezsi, and G. Langouche, Nucl. Instr. and Meth. B15, $341(1986)$.

36. Y. H. Ohtsuki, Charged Beam Interaction with Solids, Taylor and Francis-London ( 1983).

37. S. U. Campisano, G. Foti, F. Grasso, M. Lo Savio, and E. Rimini, Radiation Effects 13, 157(1972) and earlier works.

38. L. C. Feldman and B. R. Appleton, Phys. Rev. B8, 935 (1973).

39. J. A. Davies, J. Denhartog, and J. L. Whitton, Phys. Rev. 165, 345 (1968).

40. J. F. Bak, P. R. Jensen, H. Madsboll, S. P. Moller, H. E. Schiott, E. Uggerhoj, J.J. Grob, and P. Siffert, Nuclear Physics, B242, 1 (1984).

41. J. S. Forster, P. 39 in Relativistic Channeling, eds. R. A. Carrigan, Jr. and J. A. Ellison, Plenum, New York (1987).

42. K. Komaki, A. Otuka, F. Fujimoto, N. Horikawa, T. Nakanishi, G. Y. Gao, T. Iwata, S. Fukui, M. Mutou, and K. Okuno, Nucl. Instr. and Methods B2, 71 (1984).

43. D. I. Adejshvili, G. B. Bochek, V. I. Vit'ko, G. D. Kovalenko, B. I. Shramenko, Rad. Eff. Let. 87, 135 $(1985)$. 


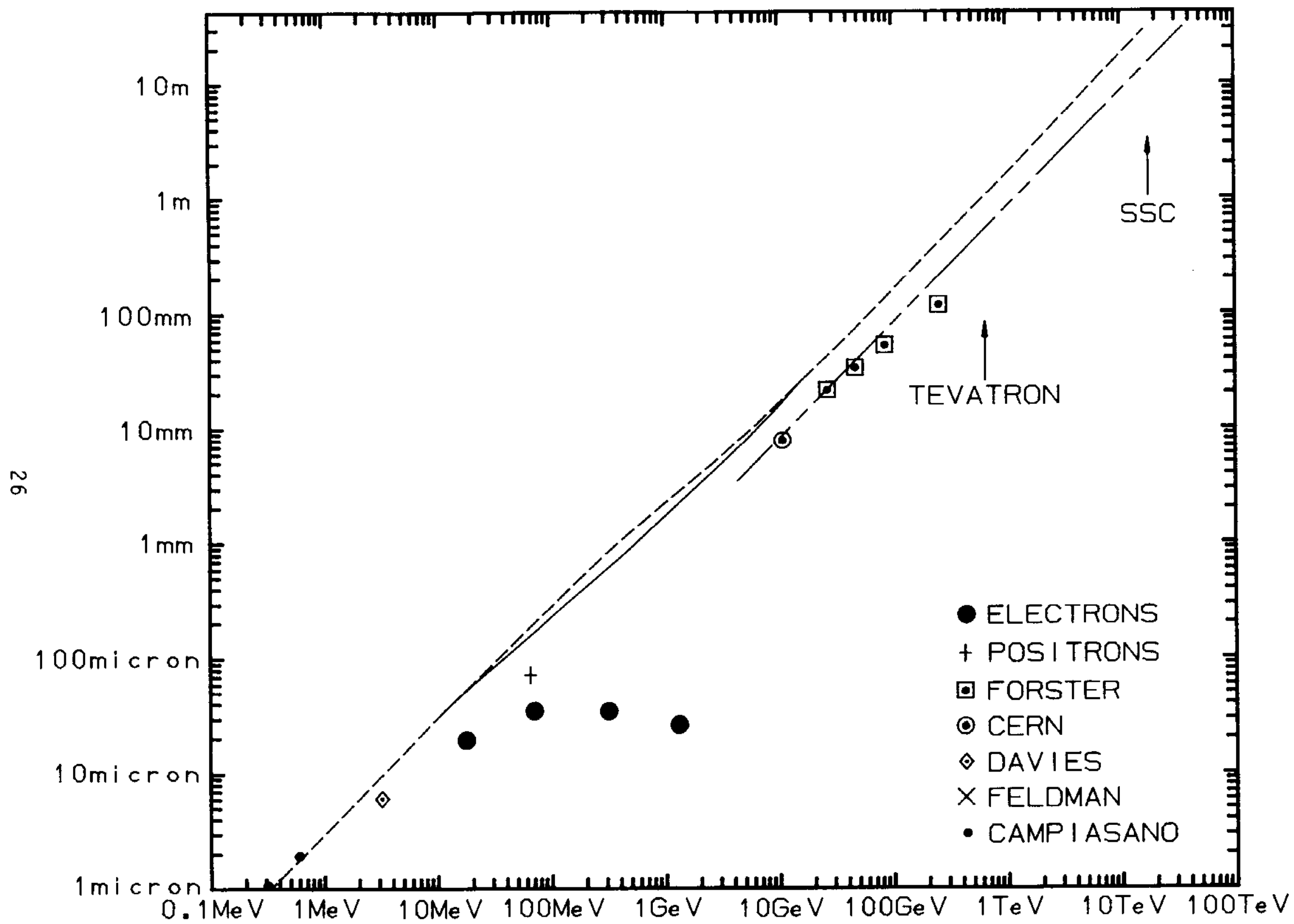

